\title{
Optimal number of routing paths in multi-path routing to minimize energy consumption in wireless sensor networks
}

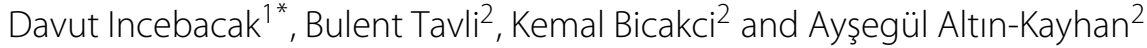

\begin{abstract}
In wireless sensor networks, multi-path routing is proposed for energy balancing which prolongs the network lifetime as compared to single-path routing where utilization of a single route between a source node and the base station results in imbalanced energy dissipation. While it is evident that increasing the number of routing paths mitigates the problem of energy over-utilization in a subset of nodes acting as relays, the net effect of the proliferation of multiple routing paths on energy balancing remains unclear. It is imperative to keep the number of routing paths as low as possible without significantly deteriorating the network lifetime; therefore, determination of the optimal number of routing paths in multi-path routing by considering the tradeoff in routing complexity and network lifetime extension is an interesting research problem. In this study, to investigate the impact of the number of routing paths in multi-path routing on network-wide energy balancing under optimal operating conditions, we build a novel mixed integer programming framework. We explore the parameter space consisting of a number of paths, number of nodes, maximum transmission range, network area, and network topology. The results of the analysis show that by utilizing the optimization scheme proposed, it is possible to achieve near-optimal energy consumption (within 1.0\% neighborhood of the case where no restrictions are imposed on the number of routing paths in multi-path routing) using at most two paths for each node.
\end{abstract}

\section{Introduction}

Optimization of energy expenditure (e.g., energy-efficient routing) is one of the most important design goals in the wireless sensor network (WSN) system design [1]. In WSNs, routing protocols discover and maintain paths between sensor nodes and the base station. The data of a sensor node is conveyed towards the base station on a single path or on multiple paths. By distributing the routing burden on multiple paths as opposed to transmitting on only a single path, multi-path routing leads to a more balanced energy dissipation in the network. There are several protocols designed for multi-path routing in WSNs [2-7]. The common goal of these protocols is to improve the energy efficiency of the network through multi-path routing. Additionally, they provide other capabilities (e.g.,

*Correspondence: idavut@metu.edu.t

${ }^{1}$ Middle East Technical University, Ankara 06800, Turkey

Full list of author information is available at the end of the article intrusion detection [2], renewable energy [3], mobile sensors [4], fault tolerance [5], quality-of-service [6], and security [7]).

Earlier studies revealed that multi-path routing improves the energy efficiency of WSNs; however, the impact of the number of paths on the level of energy efficiency is not well understood (i.e., what amount of energy efficiency improvement should be expected with each additional path?). As the number of paths increases, so does the complexity of the routing protocol. Hence, it is desirable to limit the number of paths at some point if a further increase does not improve energy efficiency significantly, yet an analysis of the impact of limiting the number of paths on energy efficiency has not been performed in the literature.

In this study, an optimization scheme to minimize the energy consumption of sensor nodes under multi-path

\section{至 Springer}

(c) 2013 Incebacak et al.: licensee Springer. This is an Open Access article distributed under the terms of the Creative Commons Attribution License (http://creativecommons.org/licenses/by/2.0), which permits unrestricted use, distribution, and reproduction in any medium, provided the original work is properly cited. 
routing is proposed. In fact, our main goal is to investigate the impact of multi-path routing on energy consumption characteristics of sensor nodes in WSNs. By developing a novel problem formulation using mathematical programming, we capture the essence of both routing and energy dissipation characteristics of multi-path routing. We analyze the impact of limiting the number of routes from the energy efficiency perspective within a general framework and without considering any specific protocol or algorithm. This approach abstracts us away from the protocol-specific overhead or implementation details. Characterization of the impact of limiting the number of routes on energy balancing in WSNs is a novel research contribution and may provide valuable insights for the design of future protocols.

There are several related concepts in this area which can also be referred as multi-path routing and may be confused with the meaning of multi-path routing as it is used in this paper. In this paper, we use the term multipath routing for partitioning the data into groups of data packets without employing data redundancy and sending each group of packets via a different path towards the base station. Alternate path routing is different from multi-path routing in the sense that a single path is used in normal operation, but alternative paths are kept ready to be used in case the primary path becomes unavailable. Redundant multi-path routing is another related term that means the data to be conveyed to the base station is transported via multiple paths with added redundancy (e.g., multiple replicas of the data are sent on different paths) [8].

Mathematical programming is a powerful tool utilized in many studies to analyze different aspects of WSNs [9]. Here, we briefly overview the previous work most relevant to our work. In [10], a mixed integer programming (MIP) framework is proposed to analyze capacity and energy consumption of IEEE 802.15.4 cluster-tree hierarchy for organizing transmissions to provide the optimal solution for the network capacity. In [11], the problem of minimizing the network cost through the minimum number of relay-station installation in continuous data-gathering WSNs is investigated by using an MIP model. In [12], an MIP-based framework for optimizing the placement of RF chargers used for energy harvesting in WSNs is proposed. In [13], two joint routing and scheduling algorithms which minimize the data delivery latency while enhancing the energy efficiency in WSNs are proposed and investigated through an MIP framework. In [14], the impact of limiting the number of incoming and outgoing links of nodes on the network lifetime is formulated as an MIP problem.

We introduce the MIP model for multi-path routing in Section 2. Section 3 presents the results of numerical analysis. Conclusions are drawn in Section 4.

\section{Model}

In our framework, we make the following assumptions:

1. The network consists of stationary nodes (both sensor nodes and the base station).

2. For a substantial amount of time (epochs), topology discovery and route creation operations are not repeated [1].

3. Network reorganization period is long enough; therefore, the energy costs of topology discovery and route creation operations constitute a small fraction (e.g., less than 1.0\% [15]) of the total network energy dissipation. Hence, routing overhead can be neglected in stationary WSNs without leading to significant underestimation of total energy dissipation.

4. A time division multiple access (TDMA)-based media access control (MAC) layer is in operation which mitigates interference between active links through a time slot assignment algorithm which outputs a conflict-free transmission schedule. A combinatorial interference model can be used to model interference, and scheduling constraints can then be modeled by a conflict graph $[16,17]$. In [18], it is shown that such an algorithm is possible; hence, collision-free communication is achieved if sufficient bandwidth requirements are satisfied. In fact, in our model, we use a modified version of the sufficient condition presented in [18]. Furthermore, it is also possible to reduce data packet collisions to negligible levels in practical MAC protocols designed with a dynamic TDMA approach $[19,20]$.

5. Energy dissipation for idle listening or overhearing in promiscuous mode is negligible. There are many intelligently designed MAC protocols for wireless networks that avoid energy waste in these modes $[19,21]$. We assume such a MAC layer is used in our framework.

6. The transmission energy model is such that the bit error rate (BER) is constant and the same for all links [22-24].

7. Energy dissipation for transmission and reception is only for data packets. The framework described in this paper can be easily extended to model the energy consumption for acknowledgement (ACK) packets as well; however, in general, the size of a typical ACK packet is much smaller than the size of data packets. Therefore, we assume that the receiver is informed of correct or incorrect reception of a data packet through an ideal feedback channel [22,25,26]. Furthermore, data packets are treated as indivisible data units (i.e., data packets are neither fragmented nor combined with other data packets until they reach the base station). 
In our network model, there is a single base station and there are $N$ sensor nodes in the network. Time is organized into rounds with duration $T_{r n d}$, and the total number of rounds is $M_{\text {rnd }}$. Each sensor node- $i$ creates the same number of data packets $\left(s_{i}\right)$ with packet length $L_{P}$ bits at each round to be conveyed to the base station (i.e., sensor nodes create CBR flows). The network topology is represented by a directed graph, $G=(V, A)$, where $V$ is the set of all nodes including the base station as node0 and $A=\left\{(i, j): i \in W, j \in V-i, d_{i j} \leq R_{\max }\right\}$ is the ordered set of arcs. We also define set $W$ which includes all nodes except node-0 (i.e., $W=V \backslash\{0\}$ ). Note that we use the expression 'node- $i$ ' when it is used in a sentence; however, we use only ' $i$ ' when it is used within a mathematical expression or when we need to refer to the indices of mathematical expressions in the narrative. The definition of $A$ implies that no node sends data to itself or to a node that is separated from it beyond the maximum transmission range $R_{\max }$. The distance between node- $i$ and node- $j$ is denoted by $d_{i j}$. Each node can forward its generated data towards the base station using at most $N_{P}$ paths. Different paths can be either disjoint or braided (i.e., two paths can share common links in their chains of links forming paths). Data generated at node- $k$ forwarded on the $l$ th path flowing from node- $i$ to node- $j$ is represented as $f_{i j}^{k l}$. Moreover, $b_{k}^{l}$ is the total amount of data packets generated by node- $k(k \in W)$ and transmitted on the $l$ th configuration to the base station, and $a_{i j}^{k l}$ indicates if arc $(i, j) \in A$ is used in the $l$ th routing configuration originated at node- $k(k \in W)$.

We adopt the energy model used in [27]. In this model, the amount of energy to transmit $L_{P}$ bits of data is $E_{t x, i j}\left(L_{P}\right)=L_{P}\left(\rho+\varepsilon d_{i j}^{\alpha}\right)$ and to receive $L_{P}$ bits of data is $E_{r x}\left(L_{P}\right)=L_{P} \rho$, where $\rho$ represents the energy dissipated in the electronic circuitry, $\varepsilon$ denotes the transmitter efficiency, and $\alpha$ represents the path loss exponent. Packet error rate is $\chi=\left(1-(1-\varphi)^{L_{P}}\right)$, where $\varphi$ is the BER. Each packet has to be transmitted $\lambda=1 /(1-\chi)$ times (average packet retransmission rate), on the average, for successful delivery of the packets. The interference range of a transmission from node- $i$ to node- $j$ is $\gamma d_{i j}$, where $\gamma$ is the interference range multiplier [18]. To model interference between links, we define a binary interference matrix, $I_{j m}^{i}$, presented in Equation 1. If node- $i$ is in the interference region of the transmission from node- $j$ to node- $m$ (i.e., $\gamma d_{j m} \geq d_{j i}$ ), then node- $i$ is blocked from receiving any data because any such flow to node- $i$ results in a conflict (packet collision). Therefore, if $I_{j m}^{i}=1$, then node- $i$ has a conflict with the flow on arc $(j, m)$ (node- $i$ is sharing the bandwidth with the flow on $\operatorname{arc}(j, m))$. On the other hand, if $I_{j m}^{i}=0$, then flow on arc $(j, m)$ is not conflicting with node- $i$. Generally speaking, the interference range is equal to or greater than the transmission range (i.e., $\gamma \geq 1$ ) $[18,28]$. This means that depending on the value of $\gamma$, node-j's transmission to node- $m$ can interfere with node$i$ even if the distance between node- $j$ and node- $m$ is less than the distance between node- $j$ and node- $i$.

$$
I_{j m}^{i}=\left\{\begin{array}{l}
1 \text { if } \gamma d_{j m} \geq d_{j i} \forall j \in W, \forall m \in V \backslash\{j\} \\
0 \text { otherwise }
\end{array}\right.
$$

The objective of the optimization problem is to minimize the the maximum energy requirement $(E)$ of sensor nodes. The network flow is modeled in the form of a series of constraints presented in the MIP model for multi-path routing (below). All system variables with their acronyms and descriptions are presented in Table 1.

\section{Table 1 Terminology for MIP formulation}

\begin{tabular}{|c|c|}
\hline Variable & Description \\
\hline$N$ & Number of nodes \\
\hline$f_{i j}^{k l}$ & $\begin{array}{l}\text { Number of data packets generated at node- } k \text { forwarded on } \\
\text { the } ~^{\text {th }} \text { path flowing from node- } i \text { to node- } j\end{array}$ \\
\hline$s_{i}$ & Number of data packets generated at node- $i$ \\
\hline$E_{r X}\left(L_{p}\right)$ & Energy consumption for receiving $L_{p}$ bits of data \\
\hline$E_{t x, i j}\left(L_{p}\right)$ & $\begin{array}{l}\text { Energy consumption for transmitting } L_{p} \text { bits of data from } \\
\text { node- } i \text { to node- } j\end{array}$ \\
\hline$d_{i j}$ & Distance between node- $i$ and node- $j$ \\
\hline$\rho$ & Energy dissipated in the electronic circuitry \\
\hline$\varepsilon$ & Transmitter efficiency \\
\hline$\alpha$ & Path loss exponent \\
\hline$E$ & Battery energy of each sensor node \\
\hline$G=(V, A)$ & Directed graph that represents network topology \\
\hline V & Set of nodes, including the base station as node- 0 \\
\hline$W$ & Set of nodes, except the base station (node-0) \\
\hline$A$ & Set of edges (links) \\
\hline 1 & Set of paths \\
\hline$a_{i j}^{k l}$ & $\begin{array}{l}\text { Binary variable to determine if arc }(i, j) \in A \text { is used in the } \\
\text { Ith routing configuration originated at sensor } k \in W \text {. }\end{array}$ \\
\hline$b_{k}^{l}$ & $\begin{array}{l}\text { Total amount of data sensed by sensor } k \in W \text { and } \\
\text { transmitted on the /th configuration to the base station }\end{array}$ \\
\hline$R$ & Radius of deployment area \\
\hline$R_{\max }$ & Maximum transmission range \\
\hline$N_{p}$ & Number of paths \\
\hline$L_{p}$ & Packet length in bits \\
\hline Mrnd & Number of rounds \\
\hline$T_{\text {rnd }}$ & Round duration \\
\hline$\xi$ & Channel data rate (bits/s) \\
\hline$\gamma$ & Interference range multiplier \\
\hline$\varphi$ & BER (bit error rate) \\
\hline$\chi$ & Packet error rate \\
\hline$\lambda$ & Average packet retransmission rate \\
\hline
\end{tabular}


Minimize $E$

Subject to:

$$
\begin{gathered}
\lambda \sum_{l=1}^{N_{P}} \sum_{k \in W}\left(\sum_{(i, j) \in A} E_{t x, i j}\left(L_{P}\right) f_{i j}^{k l}+\sum_{(j, i) \in A} E_{r x}\left(L_{P}\right) f_{j i}^{k l}\right) \\
\leq E \forall i \in W \\
\sum_{(i, j) \in A} f_{i j}^{k l}-\sum_{(j, i) \in A} f_{j i}^{k l}=\left\{\begin{array}{c}
b_{k}^{l} i=k \\
-b_{k}^{l} i=0 \\
0 \text { otherwise }
\end{array} \quad \forall i \in V, k \in W, l=1, . ., N_{P}\right.
\end{gathered}
$$

$$
\begin{aligned}
& \sum_{(j, k) \in A} f_{j k}^{k l}=0 \forall k \in W, l=1, \ldots, N_{P} \\
& \sum_{l=1}^{N_{P}} b_{k}^{l}=s_{k} M_{r n d} \forall k \in W \\
& f_{i j}^{k l} \leq s_{k} M_{r n d} a_{i j}^{k l} \forall(i, j) \in A, k \in W, l=1, . ., N_{P}
\end{aligned}
$$

$\sum_{(i, j) \in A} a_{i j}^{k l} \leq 1 \forall i, k \in W, l=1, . ., N_{P}$

$$
\sum_{(j, 0) \in A} f_{j 0}^{k l+1} \leq \sum_{(j, 0) \in A} f_{j 0}^{k l} \forall k \in W, l=1, . ., N_{P}-1
$$

$$
\begin{aligned}
& \lambda \frac{L_{P}}{\xi} \sum_{l=1}^{N_{P}} \sum_{k \in W}\left(\sum_{(i, j) \in A} f_{i j}^{k l}+\sum_{(j, i) \in A} f_{i j}^{k l}+\sum_{(j, m) \in A \backslash\{i\}} f_{j m}^{k l} I_{j m}^{i}\right) \\
& \quad \leq M_{\text {rnd }} T_{\text {rnd }} \forall i \in V
\end{aligned}
$$

$$
\begin{aligned}
& f_{i j}^{k l} \geq 0 \forall(i, j) \in A, k \in W, l=1, . ., N_{P} \\
& a_{i j}^{k l} \in\{0,1\} \forall(i, j) \in A, k \in W, l=1, . ., N_{P} \\
& b_{k}^{l} \geq 0 \forall k \in W, l=1, . ., N_{P}
\end{aligned}
$$

Constraint (2) limits the energy used by each sensor node for data transmission and reception by the total battery energy allocated to it. In fact, the objective is to minimize the energy dissipation of the maximum energy dissipating sensor node. The expression in the parenthesis gives the energy dissipation of node- $i$ on packet transmission and reception for conveying source node- $k$ 's data on its $l$ th routing path. Summation over $k$ and $l$ gives the total energy dissipation of node- $i$. Energy dissipation for retransmissions are incorporated into the model through the multiplication of the whole expression by $\lambda$. If there is no retransmission, then $\lambda=1$. The energy model we used [27] enables the adjustment of transmission energy for each node pair to enable a uniform signal-to-noise ratio (SNR) at each receiver.
Constraint (3) is known as the flow conservation constraint, which is satisfied for all $i$ (all nodes including the base station), $k$ (sensor nodes), and $l$ (routing paths). If node- $i$ is the source node $(i=k)$, then the difference between the sum of outgoing flows and the sum of incoming flows is the total amount of packets injected into the network by source node- $k$ on its $l$ th routing path $\left(b_{k}^{l}\right)$. If $i=0$ (the base station), then the all packets generated at each node- $k$ and transmitted on path- $l\left(b_{k}^{l}\right)$ reach the base station. If $i \neq k$ and $i \neq 0$, then the sum of incoming flows is equal to the sum of outgoing flows (node- $i$ is a relay node for source node- $k$ 's flow on its $l$ th path). In summary, constraint (3) ensures that all flow generated at each node- $k$ and transmitted on path- $l$ reach the base station.

Constraint (4) ensures that data generated at sensor node- $k$ and routed out to the rest of the network does not loop back to node- $k$. In other words, the sum of flows generated at node- $k$ and received by node- $k$ itself is 0 . Note that constraint (10) ensures that all flows are non-negative; hence, constraint (4) together with constraint (10) dictates that the value of any flow creating any possible loop is exactly 0 .

Constraint (5) guarantees that each node- $k(k \in W)$ generates and sends exactly a total of $s_{k} M_{r n d}$ packets to the base station. The total amount of data packets generated at node- $k$ is routed to the base station by using at most $N_{P}$ paths, and the amount of data injected by node$k$ into each one of the paths is denoted as $b_{k}^{l}$; hence, the summation over $l$ for each $k$ gives the total amount of data generated at node- $k$. Since both the flows and the amount of data injected on each path are integer variables, the packets cannot be split (all packets are created as $L_{P}$ bits long and reach the base station with the same length as they are formed). However, different paths can be used in a periodic time interleaved fashion. It is also possible that different paths are used to convey data in an aperiodic sequential arrangement. For example, if $s_{k}=1$ packet, $M_{\text {rnd }}=3,600$ rounds, $N_{P}=3$ paths, $b_{5}^{1}=1,800$ packets, $b_{5}^{2}=1,200$ packets, and $b_{5}^{3}=600$ packets, then node- 5 can create a cyclic structure with a length of six rounds. At each cycle of six rounds, three data packets, two data packets, and one data packet are conveyed to the base station using the first path, the second path, and the third path, respectively. Alternatively, node- 5 can convey all its data from round 1 to round 1,800 on its first path, from round 1,801 to round 3,000 on its second path, and from round 3,001 to round 3,600 on its third path. In our model, we do not impose any timing restriction on scheduling. We determine the optimal paths and the amount of data transported on each path throughout the entire network operation as specified by $N_{P}, s_{k}$, $M_{\text {rnd }}$, and other parameters. In fact, all feasible schedules that do not violate flow constraints in our model are equivalent. 
Constraint (6) ensures that an $\operatorname{arc}(i, j) \in A$ is marked as used for conveying data generated at node- $k$ on its $l$ th path only if there is positive flow on $(i, j) \in A\left(a_{i j}^{k l}=1\right.$ if $\left.f_{i j}^{k l}>0\right)$. Note that the value of $f_{i j}^{k l}$ can at most be $s_{k} M_{r n d}$. Such a case happens if node- $k$ uses only one routing path (i.e., $b_{k}^{1}=f_{i j}^{k 1}$ and $b_{k}^{m}=0$ for $\left.m>1\right)$. If $f_{i j}^{k l}=0$, then binary variable $a_{i j}^{k l}$ can be either 1 or 0 (i.e., by itself constraint (6) does not force $a_{i j}^{k l}$ to neither 1 nor 0 for $f_{i j}^{k l}=0$ ). However, constraint (7) as described below forces $a_{i j}^{k l}$ to be 0 if both options are feasible. Hence, constraint (6) in conjunction with constraint (7)results in $a_{i j}^{k l}=0$ if $f_{i j}^{k l}=0$.

The flow on each configuration is guaranteed to be nonbifurcated by constraint (7). Note that constraint (7) must be satisfied for all values of $i, k$, and $l$. Consider one of the possible combinations: $(i, k, l)=(3,3,1)$. For this example, constraint (7) states that data generated at node-3 which is designated to flow on its first routing path transmitted by node- 3 (the first hop of the path) can have only one receiver (i.e., there should be only one second hop node, which can be the base station or another node acting as a relay). The summation over arc set $(3, j)$ guarantees that only one of the arcs $(3, j)$ have non-zero flow because the sum is equal to or less than 1 . In the same example, assume that $j=7$ (i.e., $f_{37}^{31}=b_{3}^{1}$ and $f_{3 m}^{31}=0$ for all $m \neq 7)$. As the second hop relay, node-7 can transmit the data it received from node- $3\left(f_{37}^{31}\right)$ to only one of its neighbors (dictated again by constraint (7)). If the third hop relay is node- 8 , then $f_{78}^{31}=f_{37}^{31}=b_{3}^{1}$ and $f_{7 m}^{31}=0$ for all $m \neq 8$. Continuing in this manner, data injected by source node- 3 to its first path reaches the base station without being split into multiple branches. In other words, constraint (7) enables the construction of an unbroken and non-branching logical pipe (path) from the source to the base station for transportation of data. Indeed, $N_{P}$ is the upper limit on the number of such pipes for each source node. The maximum number of such pipes in a network of $M$ sensor nodes can be $M N_{P}$.

Constraint (8) is used to have a logical ordering of the configurations for originator nodes. Constraint (8) implies that $b_{k}^{1} \geq b_{k}^{2} \geq b_{k}^{3} \geq \ldots \geq b_{k}^{N_{P}}$ (i.e., the number of packets conveyed on the $l$ th path of source node- $k$ is greater than or equal to the number of packets conveyed on its $(l+1)$ th path).

To address bandwidth limitations in a broadcast medium, we need to make sure that the bandwidth used to transmit and receive at each node is limited by the available channel bandwidth. Such a constraint should take the shared capacity into consideration. For node- $i$, we refer to the flows around node- $i$ which are not flowing into or flowing out of node- $i$ but affecting the available bandwidth available to node- $i$ as interfering flows. Constraint (9) guarantees that for each node (including the base station), the aggregate amount of incoming flows, outgoing flows, and interfering flows can be scheduled within the given time frame $\left(T_{r n d}\right.$ seconds/round $\times M_{r n d}$ rounds $=T_{r n d} M_{r n d}$ seconds). The summation over $l, k,(i, j)$, and $(j, m)$ gives the total number of packets sharing the capacity of node- $i$. Multiplication by $L_{P}$ (bits/packet) converts the number of packets to number of bits. Division by $\xi$ (channel data rate - bits/second) transforms number of bits to seconds. Scaling with $\lambda$ is for the extra time needed due to retransmissions. This constraint is a modified version of the sufficient condition given in [18]. We note that in the numerical analysis, we choose the parameters affecting constraint (9) in such a way that the maximum value of the left-hand side of the inequality is more than an order of magnitude lower than the right-hand side value; therefore, construction of a conflict-free transmission schedule through a non-complicated time-slot assignment algorithm is possible. It is also shown that well-designed carrier sense multiple access (CSMA)-based MAC protocols are highly successful in reducing the collision rate to negligible levels provided that the network traffic is much lower (e.g., an order of magnitude) than the available capacity $[29,30]$.

Finally, constraint (10), constraint (11), and constraint (12) are nonnegativity constraints for the variables of the model.

The objective is to minimize $E$, which is the energy of the battery in each node. Once the parameter $N_{P}$ is set, the solution of the model gives the set of paths each node uses to forward its data and the amount of data transported on each of these paths in a way that the energy required by the most energy consuming node is minimized. As a result, all nodes transmit their data in order to keep the required battery energy per sensor node as low as possible. In other words, all nodes dissipate their energies in the most balanced fashion. Sensor nodes are not required to use exactly $N_{P}$ paths (e.g., it is possible for a node to use only two paths for transporting all its generated data even if $N_{P}>2$ ). Furthermore, the amount of data flow on each path $\left(b_{k}^{l}\right)$ is also determined by the MIP framework to optimize energy dissipation.

\section{Analysis}

We use the General Algebraic Modeling System (GAMS) [31] for the numerical analysis of the MIP model. GAMS consists of high-performance solvers for solving MIP models efficiently, each of which improves upon the basic approach in different ways to attain an increased solution performance. Hence, when we solve our MIP model using GAMS, one of these solvers is used to obtain the best solution. Specific implementation details are beyond the scope of this study. We should mention that we could still use GAMS if we were to solve linear programming (LP) models rather than MIP models. 
As we have mentioned, general MIP models are computationally difficult problems. They are in NP-hard class according to their computational complexity [32]. Although there are some MIP problems with efficient optimization property (i.e., they can be solved relatively easier due to their special structures), we are not aware of any previous result on the applicability of such a property in our problem. Our preliminary tests show that the LP relaxation of the problem obtained by relaxing the integrality constraints on binary variables does not provide the same optimal solution with the original MIP model and the gap is actually significant in many cases. Moreover, the solution times increase significantly as the instance sizes get larger. Hence, even for medium-sized instances, the solution times can be quite high, which can be mitigated using several implementation heuristics. Since our motivation in this paper is to explore the impact of multi-path routing rather than developing specialized efficient solution algorithms for the problem, we accept solutions with a relative gap of no more than $1.0 \%$.

Suppose that we have a solution satisfying all integer requirements and have the best objective function value $\left(z^{B}\right)$ found so far. Then, the relative gap for this solution measures the distance between $z^{B}$ and the available best bound for the optimal objective function value $\left(z^{L}\right)$ using the ratio $\frac{\left|z^{B}-z^{L}\right|}{z^{L}}$. LP-based branch-and-bound algorithms are used for solving MIPs in GAMS [31]; thus, $z^{L}$ is the LP relaxation solution of the MIP problem under consideration. Note that, in general, $z^{L}$ is not a feasible solution because integer variables are treated as continuous variables (i.e., the occurrence of non-integer values for binary variables is allowed in $z^{L}$ ). The acceptable relative gap can be controlled via the parameter optcr in GAMS, and when it is set to 0.0 , the solution algorithm stops with the exact optimal solution. In this study, we let optcr $=1.0 \%$, which provides significant time savings in exchange for an immaterial sacrifice for optimality.

In our analysis, we investigate two deployment scenarios: (1) linear deployment in which nodes are deployed equidistantly on a line without any randomness and (2) uniform random distribution in which $N$ nodes are deployed in a disc of radius $R$. There are many applications for linear sensor network deployments including border surveillance, highway traffic monitoring, safeguarding railway tracks, oil and natural gas pipeline protection, structural monitoring, and surveillance of bridges and long hallways [33]. We assume that there is a single base station located at one end in linear deployments and at the center in disc deployments. The communication parameters are chosen as $\varepsilon=100 \mathrm{pJ}, \rho=50 \mathrm{~nJ}$, and $\alpha=2$, the same as the ones in [27]. For random deployment scenarios, each problem is solved for 100 random topologies, and the results are averaged. The parameters used in the analysis are presented in Table 2.

A small-scale WSN topology is presented in Figure 1 to illustrate the network dynamics clearly. We prefer line topology to avoid more complex flow patterns in Figure 1. The numbers on each arc show the fraction of the total amount of data generated at each sensor node. For example, $f_{50}^{53}=0.23$ shows that $f_{50}^{53}=0.23 \times \lambda \times s_{5} \times$ $M_{\text {rnd }}$ packets, which is equal to 1,010 packets; furthermore, it also shows that $b_{5}^{1}=1,010$ packets. Nodes are placed on a line with $30 \mathrm{~m}$ separation and $R_{\max }=150 \mathrm{~m}$. Base station is node- 0 . The MIP model is solved with (a) $N_{P} \rightarrow \infty$, (b) $N_{P}=2$, and (c) $N_{P}=1$. Energy dissipations are indicated near the nodes. When there is no limit on the number of paths used by each sensor node $\left(N_{P} \rightarrow \infty\right)$, the required battery energy for each node is $5.86 \mathrm{~J}$ (i.e., energy dissipations of all nodes are exactly balanced). For the optimal case, node- 4 and node- 5 use three paths and other nodes use a single path. When the number of paths used by each sensor node is upper limited by $2\left(N_{P}=2\right)$, the required energy for each sensor node becomes $5.87 \mathrm{~J}$ (i.e., percentage energy overhead is $0.14 \%$ with respect to the $N_{P} \rightarrow \infty$ case). Note that all sensor nodes spend the same amount of energy; however, energy dissipation is slightly higher than the $N_{P} \rightarrow \infty$ case due to the suboptimal path selection.

For the case of $N_{P}=1$ (i.e., single-path routing), energy overhead becomes $21.30 \%$ when compared to the $N_{P} \rightarrow$

\section{Table 2 Parameters used in analysis}

\begin{tabular}{ll}
\hline Parameter & Values \\
\hline$\rho$ & $50 \mathrm{~nJ} / \mathrm{bit}$ \\
$\varepsilon$ & $100 \mathrm{pJ} / \mathrm{bit} / \mathrm{m}^{2}$ \\
$\alpha$ & 2 \\
$N$ & $5-50$ \\
Deployment scenarios & Linear (1-D) equidistant, disc (2-D) random \\
Inter-node distance (1-D) & $30 \mathrm{~m}, 10 \mathrm{~m}$ \\
Network radius R (2-D) & $100 \mathrm{~m}-1,600 \mathrm{~m}$ \\
$s_{i}$ & $1 \mathrm{packet}$ \\
$N_{p}$ & $1-\infty$ \\
$R_{\text {max }}$ & $R-R / 2$ \\
$L p$ & $2048 \mathrm{bits}$ (256 Bytes) \\
$M_{\text {rnd }}$ & $3600 \mathrm{rounds}$ \\
$T_{\text {rnd }}$ & $60 \mathrm{~s}$ \\
$\xi$ & $256 \mathrm{Kbps}$ \\
$\gamma$ & 1.7 \\
$\varphi$ & $10^{-4}$ \\
$\chi$ & 0.18 \\
$\lambda$ & 1.22 \\
\hline &
\end{tabular}




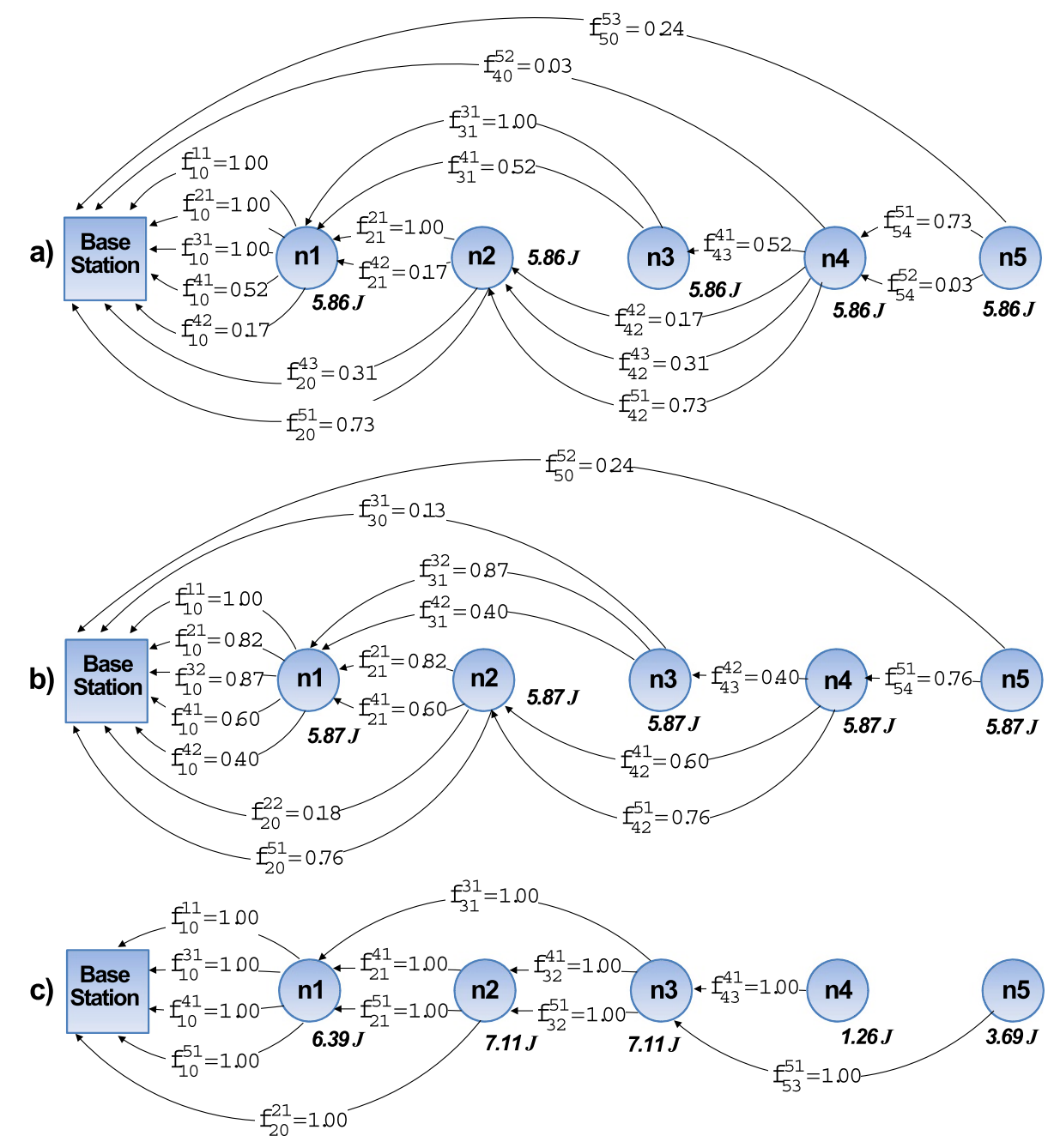

Figure 1 Optimal flows that minimize energy dissipation in (one dimensional) linear topology. The numbers on each arc show the fraction of the total amount of data generated at each sensor node. For example, $f_{50}^{53}=0.23$ shows that $f_{50}^{53}=0.23 \times \lambda \times s_{5} \times M_{\text {rnd }}$ packets, which is equal to 1,010 packets; furthermore, it also shows that $b_{5}^{1}=1,010$ packets. Nodes are placed on a line with $30 \mathrm{~m}$ separation and $R_{\max }=150 \mathrm{~m}$. Base station is node- 0 . The MIP model is solved with (a) $N_{P} \rightarrow \infty$, (b) $N_{P}=2$, and (c) $N_{P}=1$. Energy dissipations are indicated near the nodes.

$\infty$ case - the energy requirement for the maximum energy dissipating node (node-2) is $7.11 \mathrm{~J}$. Unlike $N_{P} \rightarrow \infty$ and $N_{P}=2$ cases, in the $N_{P}=1$ case, sensor nodes do not spend an equal amount of energy (e.g., $6.39 \mathrm{~J}$ for node-1 and $7.11 \mathrm{~J}$ for node-2). Hence, we observe that singlepath routing cannot lead to a balanced energy dissipation regime in the network, which leads to over-utilization of some nodes' batteries. We investigate line topologies by varying the number of sensor nodes and inter-node distance to confirm the effects of limiting the number of routing paths observed in the small scale line topology in Figure 1 which also holds for larger line topologies with different inter-node separation values.

In Figure 2, energy overhead (with respect to the $N_{P} \rightarrow \infty$ case) as a function of inter-node separation is presented for linear topology and for $N_{P}=1$ and $N_{P}=2$ cases with the number of nodes ranging from 20 nodes to 50 nodes. All nodes can transmit to and receive from any other node in the network because nodes' transmission ranges are not limited in this scenario (i.e., $R_{\max } \rightarrow \infty$ ). Energy overhead values of all $N_{P}=2$ curves are always less than $1.00 \%$. On the other hand, energy overhead of single-path routing stays in the $5.58 \%$ to $11.52 \%$ band.

In Figure 3, the impact of $R_{\max }$ on energy overhead in linear topology with 40 nodes for $N_{P}=1$ and $N_{P}=2$ is presented. Energy overhead values of all $N_{P}=2$ curves are less than $1.00 \%$. On the other hand, energy overhead of single-path routing stays in the $1.22 \%$ to $7.48 \%$ band. In single-path routing $\left(N_{P}=1\right)$, energy overhead is lower for lower $R_{\max }$ because for lower $R_{\max }$, the $N_{P} \rightarrow \infty$ case is 


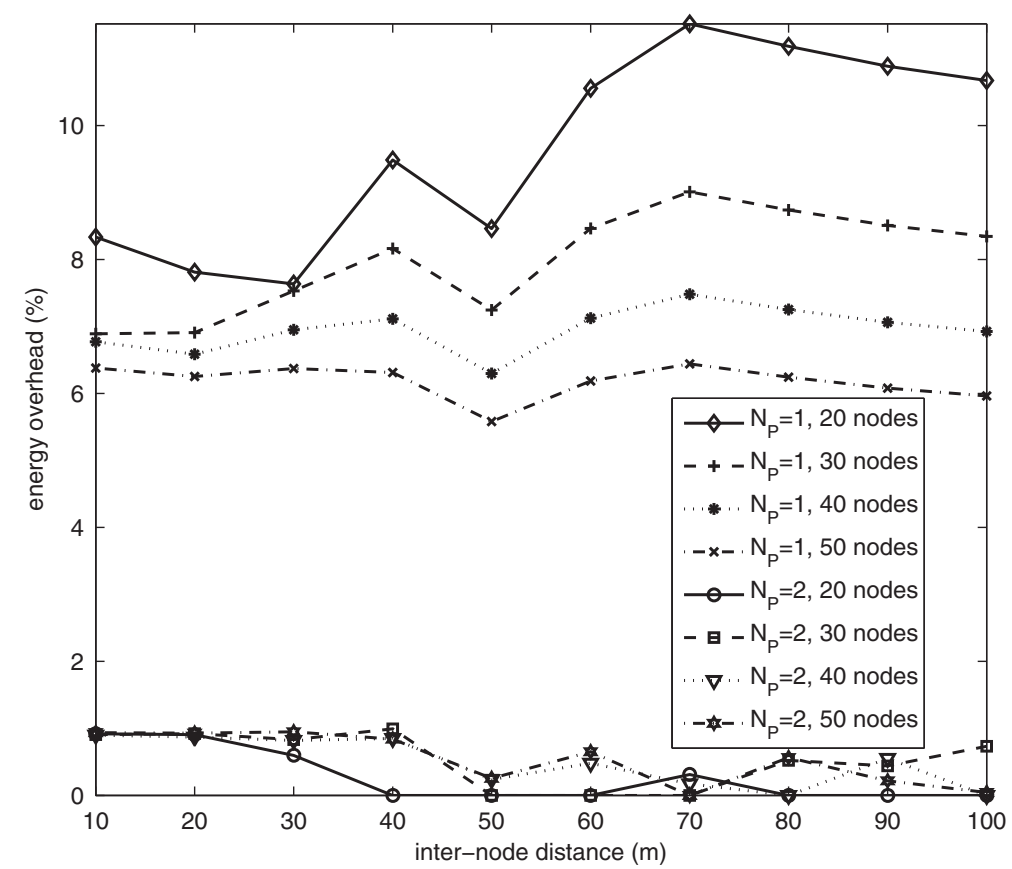

Figure 2 Percentage energy overhead with respect to the $N_{P} \rightarrow \infty$ case in linear topology $\left(R_{\max } \rightarrow \infty\right)$.

not as effective as it is with higher $R_{\max }$ (i.e., the number of paths to choose from decreases as $R_{\max }$ decreases which narrows the options available for energy balancing).

In Figures 4 and 5, we present results on twodimensional networks to generalize our results in onedimensional networks to two-dimensional networks. In
Figures 4 and 5, energy overheads as functions of the number of sensor nodes and disc radius are presented, respectively, for different $R_{\max }$ and $N_{P}$ 's. In Figure 4 , for $N_{P}=1$, as $R_{\max }$ decreases, the energy overhead also decreases. This is because for smaller $R_{\max }$ values even with $N_{P} \rightarrow \infty$, energy balancing is not as effective as

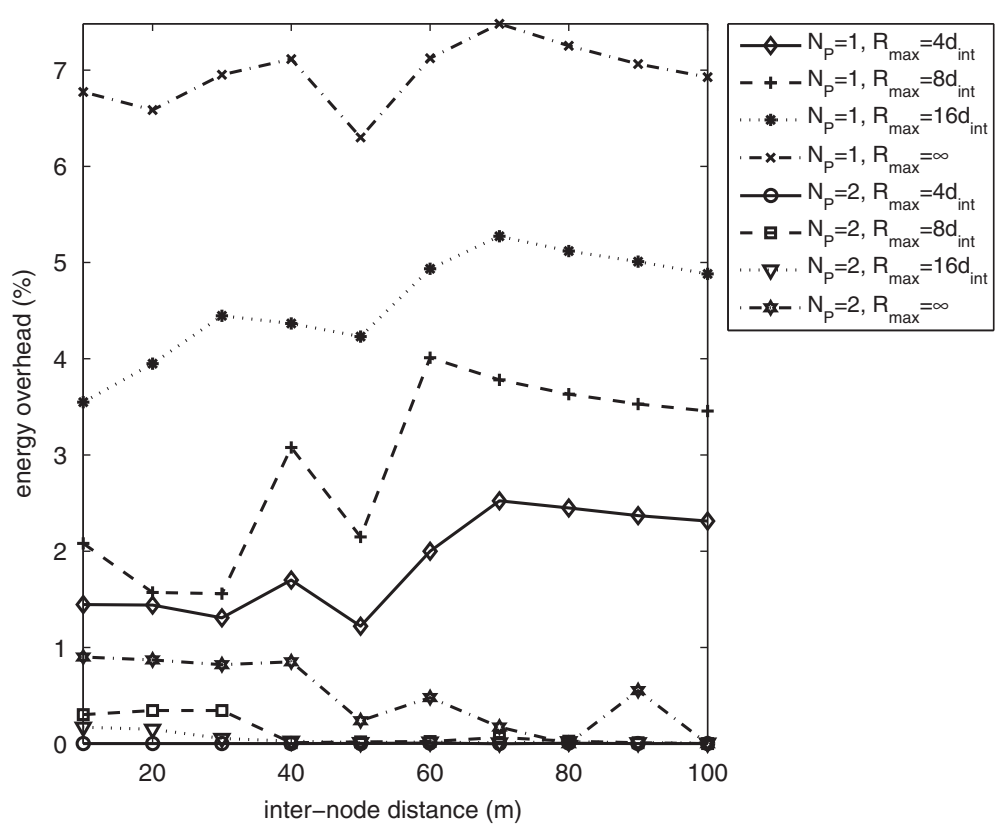

Figure 3 Percentage energy overhead with respect to the $N_{P} \rightarrow \infty$ case in linear topology $(N=40)$. 


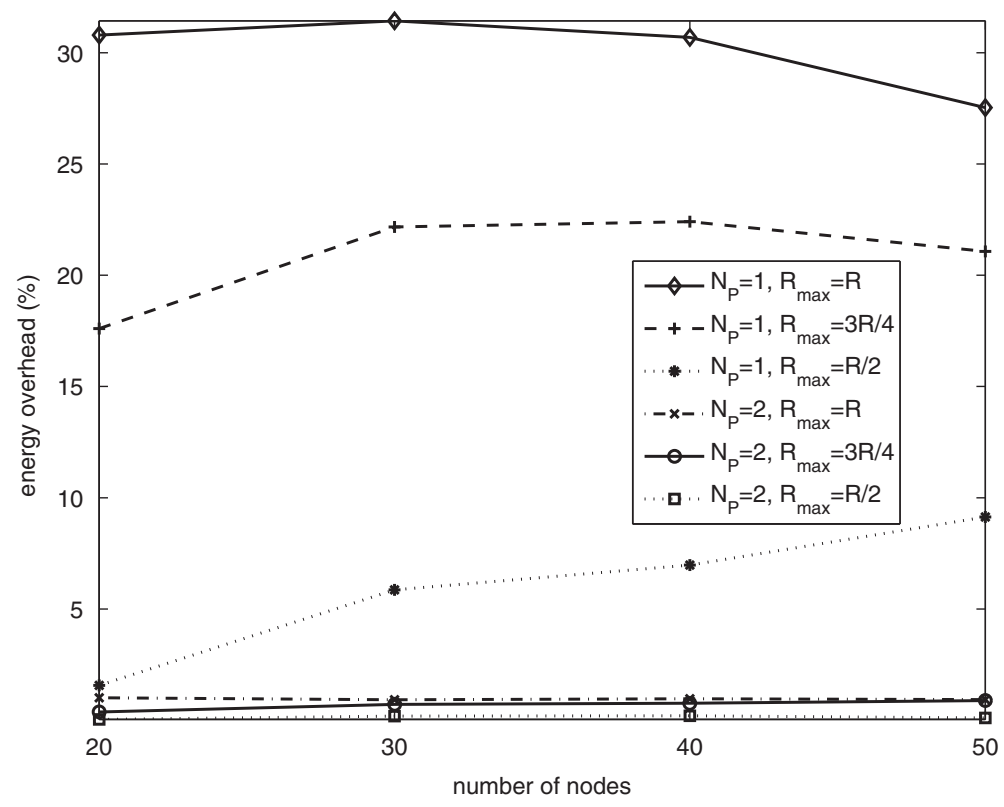

Figure 4 Percentage energy overhead with respect to the $N_{P} \rightarrow \infty$ case in disc topology with $R=200 \mathrm{~m}$.

in the case of $R_{\max } \rightarrow \infty$ due to more limited routing options. In single-path routing, energy overheads are in the $31.43 \%$ to $27.53 \%$ band and $1.57 \%$ to $9.13 \%$ band for $R_{\max }=R(200 \mathrm{~m})$ and $R_{\max }=R / 2(100 \mathrm{~m})$, respectively. The characteristics of energy overhead exhibit similar trends in Figure 5 (i.e., energy overhead is dominated by $\left.R_{\max }\right)$. Both in Figures 4 and 5 , energy overheads of all two-path routing scenarios are less than $1.00 \%$.
In all topologies explored in this study, our experiments revealed that energy overhead values for $N_{P}>2$ (not presented in the figures) are always less than $1.00 \%$.

\section{Conclusion}

In this paper, we presented an MIP framework to investigate the energy dissipation of WSNs as a function of the number of routing paths. We explored various WSN

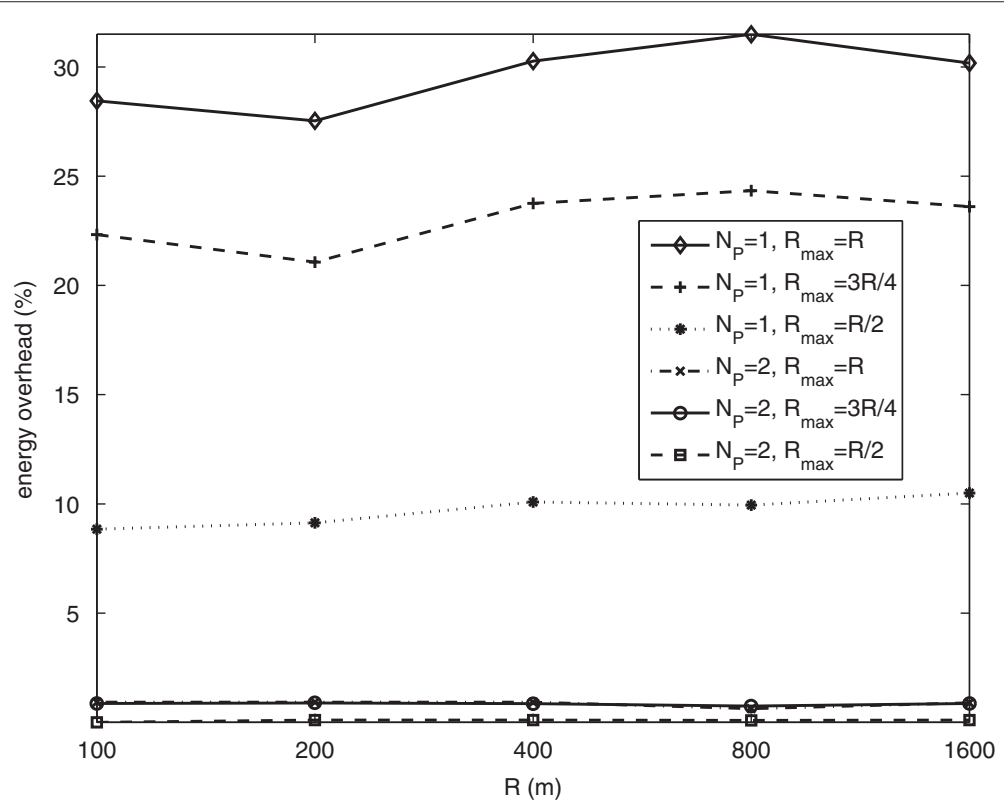

Figure 5 Percentage energy overhead with respect to the $N_{P} \rightarrow \infty$ case disc topology with 50 sensor nodes. 
scenarios in both one-dimensional and two-dimensional network topologies by sampling the parameter space through the developed model.

Our analysis revealed that single-path routing may lead to more than $30.00 \%$ energy overhead due to the lack of sufficient number of energy balancing routes. On the other hand, multi-path routing with only two paths results in near-optimal values with at most $1.00 \%$ energy overhead. Thus, our main conclusion is that use of more than two paths for energy balancing in multi-path routing for WSNs does not bring any significant benefit from an energy efficiency perspective. The MIP framework we presented in our study can easily be tailored to accommodate other aspects of multi-path routing in WSNs. Nevertheless, the concept of an end-to-end path should exist for our results to be relevant.

\section{Competing interests}

The authors declare that they have no competing interests.

\section{Author details}

${ }^{1}$ Middle East Technical University, Ankara 06800, Turkey. ${ }^{2}$ TOBB University of Economics and Technology, Ankara 06560, Turkey.

Received: 7 March 2013 Accepted: 11 October 2013

Published: 30 October 2013

\section{References}

1. K Akkaya, M Younis, A survey on routing protocols for wireless sensor networks. Ad Hoc Netw. 3, 325-349 (2005)

2. A Ouadjaout, Y Challal, N Lasla, M Bagaa, SEIF: secure and efficient intrusion-fault tolerant routing protocol for wireless sensor networks, in Proc. International Conference on Availability, Reliability and Security (ARES), (Barcelona, 4-7 March 2008), pp. 503-508

3. TKandasamy, J Krishnan, Multipath routing scheme in solar powered wireless sensor networks, in Proc. IFIP International Conference on New Technologies, Mobility and Security (NTMS), (Tangier, 5-7 Nov 2008), pp. 1-5

4. A Aronsky, A Segall, A multipath routing algorithm for mobile Wireless Sensor Networks, in Proc. IFIP Wireless and Mobile Networking Conference (WMNC), (Budapest, 13-15 Oct 2010), pp. 1-6

5. A Hadjidj, A Bouabdallah, Y Challal, HDMRP An efficient fault-tolerant multipath routing protocol for heterogeneous wireless sensor networks, in Springer Lecture Notes of the Institute for Computer Sciences, Social Informatics and Telecommunications Engineering (LNICST), Volume 74 (Springer, New York, 2011), pp. 469-482

6. H Heikalabad, SR Rasouli, F Nematy, N Rahmani, QEMPAR: QoS and energy aware multi-path routing algorithm for real-time applications in wireless sensor networks. Int. J. Comput. Sci. Issues. 8, 466-471 (2011)

7. R Gupta, H Dhadhal, Secure multipath routing in wireless sensor networks. Int. J. Electron. Comput. Sci. Eng. 1, 585-589 (2012)

8. K Bicakci, B Tavli, Denial-of-service attacks and countermeasures in IEEE 802.11 wireless networks. Comput. Stand. Interfaces. 31, 931-941 (2009)

9. F Ishmanov, AS Malik, SM Kim, Energy consumption balancing (ECB) issues and mechanisms in wireless sensor networks (WSNs), a comprehensive overview. Eur. Trans. Telecommunications. 22, 151-167 (2011)

10. F Theoleyre, B Darties, Capacity and energy-consumption optimization for the cluster-tree topology in IEEE 802.15.4. IEEE Commun. Lett. 15 816-818 (2011)

11. CPrommak, S Modhirun, Optimal wireless sensor network design for efficient energy utilization, in Proc. IEEE Workshops of International Conference on Advanced Information Networking and Applications (WAINA), (Singapore, 22-25 March 2011), pp. 814-819

12. M Erol-Kantarci, HT Mouftah, Mission-aware placement of RF-based power transmitters in wireless sensor networks, in Proc. IEEE Symposium on Computers and Communications (ISCC), (Cappadocia, 1-4 July 2012), pp. 12-17
13. DH Tran, DS Kim, Minimum latency and energy efficiency routing with lossy link awareness in wireless sensor networks, in Proc. IEEE International Workshop on Factory Communication Systems (WFCS), (Lemgo, 21-24 May 2012), pp. 75-78

14. B Tavli, MB Akgun, K Bicakci, Impact of limiting number of links on the lifetime of wireless sensor networks. IEEE Communications Letters. 15 43-45 (2011)

15. K Bicakci, H Gultekin, B Tavli, The impact of one-time energy costs on network lifetime in wireless sensor networks. IEEE Commun. Lett. 13 905-907 (2009)

16. K Jain, J Padhye, VN Padmanabhan, L Qiu, Impact of interference on multi-hop wireless network performance, in Proc. ACM Annual International Conference on Mobile Computing and Networking (MOBICOM) (ACM, New York, 2003), pp. 66-80

17. P Gupta, PR Kumar, The capacity of wireless networks. IEEE Trans. Inf Theory. 46, 388-404 (2000)

18. M Cheng, X Gong, L Cai, Joint routing and link rate allocation under bandwidth and energy constraints in sensor networks. IEEE Trans. Wireless Commun. 8, 3770-3779 (2009)

19. I Demirkol, C Ersoy, F Alagoz, MAC protocols for wireless sensor networks: a survey. IEEE Commun. Mag. 44, 115-121 (2006)

20. B Tavli, W Heinzelman, Energy and spatial reuse efficient network-wide real-time data broadcasting in mobile ad hoc networks. IEEE Trans. Mobile Comput. 5, 1297-1312 (2006)

21. B Tavli, W Heinzelman, Energy-efficient real-time multicast routing in mobile ad hoc networks. IEEE Trans. Comput. 60, 707-722 (2011)

22. R Madan, S Cui, S Lall, AJ Goldsmith, Modeling and optimization of transmission schemes in energy-constrained wireless sensor networks. IEEE/ACM Trans. Netw. 15, 1359-1372 (2007)

23. R Madan, S Cui, S Lal, A Goldsmith, Cross-layer design for lifetime maximization in interference-limited wireless sensor networks. IEEE Trans. Wireless Commun. 5, 3142-3152 (2006)

24. A Karnik, A lyer, C Rosenberg, Throughput-optimal configuration of fixed wireless networks. IEEE/ACM Trans. Netw. 16, 1161-1174 (2008)

25. Y Sankarasubramaniam, IF Akyildiz, SW McLaughlin, Energy efficiency based packet size optimization in wireless sensor networks, in Proc. IEEE International Workshop on Sensor Network Protocols and Applications (SNPA), (Anchorage, 11 May 2003), pp. 1-8

26. K Keada, M Zuniga, A Helmy, B Krishnamachari, Energy-efficient forwarding strategies for geographic routing in lossy wireless sensor networks, in Proc. International Conference on Embedded Networked Sensor Systems (SenSys) (ACM, New York, 2004), pp. 108-121

27. W Heinzelman, A Chandrakasan, H Balakrishnan, An application specific protocol architecture for wireless microsensor networks. IEEE Trans. Wireless Commun. 1, 660-670 (2002)

28. I Rhee, A Warrier, M Aia, J Min, ML Sichitiu, Z-MAC, a hybrid MAC for wireless sensor networks. IEEE/ACM Trans. Netw. 16, 511-524 (2008)

29. K Duffy, D Malone, D Leith, Modeling the 802.11 distributed coordination function in non-saturated conditions. IEEE Commun. Lett. 9, 715-717 (2005)

30. D Malone, K Duffy, D Leith, Modeling the 802.11 distributed coordination function in nonsaturated heterogeneous conditions. IEEE/ACM Trans. Netw. 15, 159-172 (2007)

31. A Brooke, D Kendrick, A Meeraus, R Raman, GAMS: A User's Guide (GAMS Development Corporation, Washington DC, 1998)

32. L Wolsey, Integer Programming (Wiley-Interscience Series in Discrete Mathematics and Optimization, Wiley, New York, 1998)

33. X Liu, P Mohapatra, Proc. International Workshop on Measurement, Modelling, and Performance Analysis of Wireless Sensor Networks (SenMetrics), (San Diego, 21 July 2005), pp. 78-85

\section{doi:10.1186/1687-1499-2013-252}

Cite this article as: Incebacak et al:: Optimal number of routing paths in multi-path routing to minimize energy consumption in wireless sensor networks. EURASIP Journal on Wireless Communications and Networking 2013 2013:252. 